University of Nebraska - Lincoln

DigitalCommons@University of Nebraska - Lincoln

March 1988

\title{
Hyperspherical Description of Two-Electron Systems
}

Anthony F. Starace

University of Nebraska-Lincoln, astarace1@unl.edu

Follow this and additional works at: https://digitalcommons.unl.edu/physicsstarace

Part of the Physics Commons

Starace, Anthony F., "Hyperspherical Description of Two-Electron Systems" (1988). Anthony F. Starace Publications. 126.

https://digitalcommons.unl.edu/physicsstarace/126

This Article is brought to you for free and open access by the Research Papers in Physics and Astronomy at DigitalCommons@University of Nebraska - Lincoln. It has been accepted for inclusion in Anthony F. Starace Publications by an authorized administrator of DigitalCommons@University of Nebraska - Lincoln. 


\title{
FrOm: FUNDAMENTAL PROCESSES OF ATOMIC DYNAMICS \\ Edited by J. S. Briggs, H. Kleinpoppen, and H. O. Lutz (Plenum Publishing Corporation, 1988)
}

\section{HYPERSPHERICAL DESCRIPTION OF TWO-ELECTRON SYSTEMS}

\author{
Anthony F. Starace \\ Department of Physics and Astronomy \\ The University of Nebraska \\ Lincoln, Nebraska 68588-0111
}

\section{INTRODUCTION}

While the use of hyperspherical coordinates to describe two-electron systems is quite old, $1-8$ it was Macek' $s^{9}$ introduction of a quasi-separable approximation in hyperspherical coordinates which made possible a host of theoretical studies elucidating the symmetries of doubly-excited states and the dynamics of processes involving two-electron atoms and ions. This work, up to about mid 1982, has been reviewed by Fano.10 However, much additional progress has been made since 1982, particularly on the symmetries of doubly excited electronic states, on the correspondences with molecular descriptions of two-electron systems, on the limitations of the quasi-separable approximation at small and at large distances, and on the hyperspherical descriptions of systems with an arbitrary number of electrons. It is the purpose of this and the next three papers, then, to present an updated review of our current theoretical understanding of electron correlations.

In this first paper we review the hyperspherical description of two electron systems, beginning with the quasi-separable approximation of Macek 9 and its applications. We next discuss the concept of two electron motion along a potential ridge as the "pathway" by which two-electron states of high excitation are realized. The need for alternative representations of two-electron wave functions near the nucleus and at distances far from the nucleus is then analyzed. Finally, a number of extensions of the quasi-separable approximation in hyperspherical coordinates to other three-particle systems are mentioned.

The following three papers in this series on the theory of electron correlations are concerned with these additional aspects: the paper of Lin discusses the symmetries of two-electron excited states, primarily based on the quasi-separable approximation in hyperspherical coordinates; the paper of Feagin discusses a molecular description of two-electron adiabatic potentials and symmetries; finally, the paper of Cavagnero discusses the hyperspherical description of $\mathrm{N}$-electron atoms and ions, with $\mathrm{N}>2$. 


\section{General Orientation}

A two electron wavefunction $\psi\left(\vec{r}_{1}, \vec{r}_{2}\right)$ is usually described by the six coordinates $r_{1}, r_{2}, \hat{r}_{1}$, and $\hat{r}_{2}$ of the two electrons. In hyperspherical coordinates the magnitudes of the individual radial coordinates, $r_{1}$ and $r_{2}$, are replaced by a hyperspherical radius, $R$, and a hyperspherical angle, $\alpha$, where

$$
\mathrm{R} \equiv\left(\mathrm{r}_{1}^{2}+\mathrm{r}_{2}^{2}\right)^{1 / 2}
$$

and

$\alpha \equiv \arctan \left(r_{2} / r_{1}\right)$

The radius $\mathrm{R}$ measures the "size" of the two electron state, while the angle $\alpha$ measures the radial correlation of the two electrons. Note that when $\alpha=\pi / 4, r_{1}=r_{2}$; when $\alpha \approx 0$ or $\approx \pi / 2$, one of the electrons is at a much larger distance from the nucleus than the other.

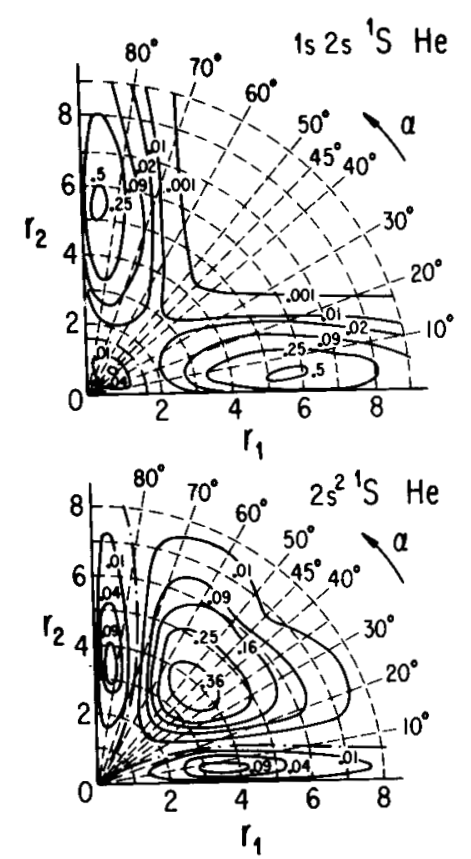

Fig. 1. Contour plot of the approximate probability distribution $\left|\psi\left(\vec{r}_{1}, \vec{r}_{2}\right)\right|^{2}$ for He. (a) $1 \mathrm{~s} 2 \mathrm{~s}^{1} \mathrm{~s}$ (b) $2 \mathrm{~s}^{2} 1 \mathrm{~S}$. Solid Lines: lines of constant probability. Dot-Dash Lines: nodal lines. (From Ref. 12)

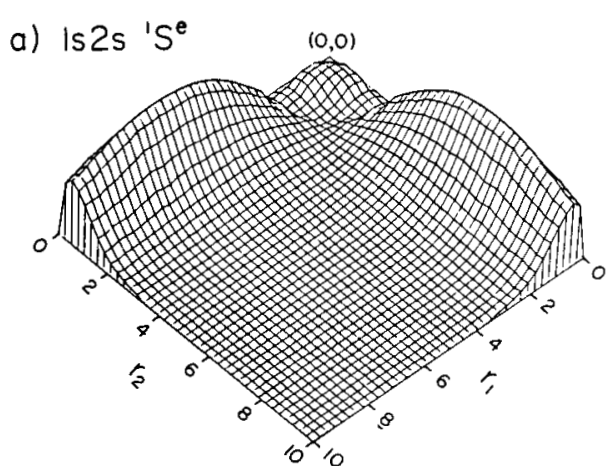

b) $2 s^{2} \cdot s^{e}$

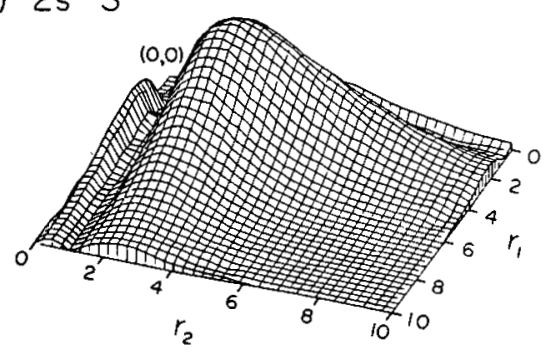

Fig. 2. Relief map of the approximate probability distribution $\left|\psi\left(\vec{r}_{1}, \vec{r}_{2}\right)\right|^{2}$ for $\mathrm{He}$. (a) $1 \mathrm{~s} 2 \mathrm{~s}^{1 \mathrm{~s}}$ (b) $2 s^{2} i_{s}$ (From Ref. 13) 
Before summarizing the features of the Schrödinger equation in these. coordinates let us look first at plots of approximate two-electron probabilities, $\left|\psi\left(R, \alpha, \hat{r}_{1}, \hat{r}_{2}\right)\right|^{2}$, in these coordinates. Fig. 1 shows contour plots 11,12 and $\mathrm{Fig} .2$ shows relief maps 13 for the probability distributions of the singly-excited state $1 \mathrm{~s}^{2} \mathrm{~s}^{1} \mathrm{~S}$ and the doubly-excited state $2 s^{2}{ }^{1} \mathrm{~S}$ of He. (Note that the wave functions are calculated in the approximation that each electron has an orbital angular momentum equal to zero in order to eliminate all dependence on the angular variables $\hat{r}_{1}$ and $\hat{r}_{2}$; since the angular dependence is trivial, these states are symmetric about $\alpha=\pi / 4$, i.e., under interchange of $r_{1}$ and $r_{2}$ ) The most obvious distinguishing features of the two probability distributions is that the one for the singly excited state is largest along $\alpha=0$ and $\alpha \approx \pi / 2$ (implying one electron is much further from the nucleus than the other) while the one for the doubly excited state is largest along $\alpha=\pi / 4$ (implying both electrons are comparably excited, i.e., $\alpha=\pi / 4$ when $r_{1}=r_{2}$ ). A second important feature is the behavior of the nodal lines for the two probability distributions. The $1 \mathrm{~s} 2 \mathrm{~s}^{1} \mathrm{~S}$ state has a single nodal line along $R \approx 2$, while the $2 s^{2} 1 \mathrm{~S}$ state has two nodal lines along $\alpha \approx$ constant: one along $5^{\circ}<\alpha<30^{\circ}$ and the other along $60^{\circ}<\alpha<85^{\circ}$. The fact that the pattern of nodal lines is approximately along the orthonormal grid of constant $R$ and constant $\alpha$ implies a quasi-separability of $R$ and $\alpha$ coordinates.

The nodal line pattern for a particular state serves also to classify the state. 13 The ground state of $\mathrm{He}, 1 \mathrm{~s}^{2} 1 \mathrm{~s}$, has a spherically symmetric probability distribution and is the first member of the singly-excited channel $1 \mathrm{sns} 1_{S}$, which converges to the $\mathrm{He}^{+}(n=1)$ threshold. The single node in $\mathrm{R}$ for the state $1 \mathrm{~s} 2 \mathrm{~s}$ is, shown in Figs. $1(\mathrm{a})$ and $2(\mathrm{a})$, characterizes it as the second member of the $1 \mathrm{sns}^{1} \mathrm{~S}$ channel. The state $2 \mathrm{~s}^{2}{ }^{1} \mathrm{~S}$, shown in Figs. 1 (b) and 2 (b), has no radial nodes. It is the first member of the Rydberg series 2 sns $1 \mathrm{~S}$ converging to the $\mathrm{He}^{+}(\mathrm{n}=2)$ threshold. The two nodes approximately along constant $\alpha$, symmetrical about $\alpha=\pi / 4$, characterize $2 \mathrm{~s}^{2}{ }^{1} \mathrm{~s}$ as a member of this second Rydberg channel. Thus nodes in $R$ characterize the excitation of a state within a channel while nodes in $\alpha$ characterize the various channels.13

\section{Two-Electron Schrödinger Equation}

In hyperspherical coordinates the non-relativistic two-electron Schrödinger equation becomes

$$
\begin{gathered}
\left(\frac{d^{2}}{d R^{2}}-\frac{1}{R^{2}}\left(-\frac{d^{2}}{d \alpha^{2}}-\frac{1}{4}+\frac{\ell_{1}^{2}}{\sin ^{2} \alpha}+\frac{l_{2}^{2}}{\cos ^{2} \alpha}\right)-\frac{C(\alpha, \theta 12)}{R}+2 E\right) \\
\quad x\left(R^{5 / 2} \sin \alpha \cos \alpha \psi\right)=0
\end{gathered}
$$

where the potential $-C\left(\alpha, \theta_{12}\right)$ is proportional to the sum of the nuclear and electrostatic potentials,

$$
\begin{aligned}
-C\left(\alpha, \theta_{12}\right) & =R\left(-\frac{2 Z}{r_{1}}-\frac{2 Z}{r_{2}}+\frac{2}{\left|\vec{r}_{2}-\vec{r}_{1}\right|}\right) \\
& =-\frac{2 Z}{\cos \alpha}-\frac{2 Z}{\sin \alpha}+\frac{2}{\left(1-\sin 2 \alpha \cos \theta_{12}\right)^{1 / 2}},
\end{aligned}
$$


$\vec{l}_{1}$ and $\vec{l}_{2}$ are the usual orbital angular momentum operators for the individual electrons, $\theta_{12} \equiv \cos ^{-1} \hat{r}_{1} \cdot \hat{r}_{2}$, and $Z$ is the nuclear charge.

In the hyperspherical coordinate method of Macek, 9 the two-electron wavefunction $\psi_{v}\left(\vec{r}_{1}, \vec{r}_{2}\right)$ is expanded in terms of a complete set of adiabatic eigenfunctions $\phi_{\mu}\left(R ; \alpha, \hat{r}_{1}, \hat{r}_{2}\right)$, which depend parametrically on the hyperspherical radius, $R$, and are functions of the five angular variables, $\alpha$, $\hat{r}_{1}$, and $\hat{r}_{2}$. The form of $\psi$ is thus:

$$
\psi_{\nu}\left(R, \alpha, \hat{r}_{1}, \hat{r}_{2}\right)=\left(R^{5 / 2} \sin \alpha \cos \alpha\right)^{-1} \sum_{\mu} F_{\mu \nu}(R) \phi_{\mu}\left(R ; \alpha ; \hat{r}_{1}, \hat{r}_{2}\right)
$$

The angular function $\phi_{\mu}$ is defined to satisfy the following differential equation in atomic units $(h=e=m=1)$ :

$$
\left(-\frac{d^{2}}{d \alpha^{2}}+\frac{l_{1}^{2}}{\cos ^{2} \alpha}+\frac{l_{2}^{2}}{\sin ^{2} \alpha}-\operatorname{RC}\left(\alpha, \theta_{12}\right)\right) \phi_{\mu}=-U_{\mu}(R) \phi_{\mu}
$$

Here $-C\left(\alpha, \theta_{12}\right)$ is defined in Eq. (4) and $U_{\mu}(R)$ is an eigenvalue which is parametrically dependent on $\mathrm{R}$. Upon substituting Eq. (5) in the two-electron Schrodinger equation and using Eq. (6), one obtains the following set of coupled differential equations for the radial functions $F_{\mu \nu}(R)$ :

$$
\begin{aligned}
& \left(\frac{d^{2}}{d R^{2}}+\frac{U_{\mu}(R)+\frac{1}{4}}{R^{2}}+\left(\phi_{\mu}, \frac{\partial^{2} \phi_{\mu}}{\partial R^{2}}\right)+2 E\right) F_{\mu \nu}(R) \\
& +\sum_{\mu^{\prime} \neq \mu}\left(\left(\phi_{\mu^{\prime}}, \frac{\partial^{2} \phi_{\mu^{\prime}}}{\partial R^{2}}\right)+2\left(\phi_{\mu^{\prime}}, \frac{\partial \phi_{\mu^{\prime}}}{\partial R}\right) \frac{\partial}{\partial R}\right) F_{\mu^{\prime} \nu^{\prime}}(R)=0
\end{aligned}
$$

In Eq. (7) the coupling matrix elements $\left(\phi_{\mu}, \partial^{n_{\phi_{\mu}}}, / \partial R^{n}\right), n=1,2$, involve integration over the five angular variables only and are thus parametrically dependent on $R$.

\section{The Quasi-Separable Approximation}

Each of the potentials $U_{\mu}(R)$ and its corresponding angular eigenfunction $\phi_{\mu}$ define a hyperspherical channel $\mu$. These channels are coupled through the radial derivative matrix elements in Eq. (7). In a quasiseparable hyperspherical (QSH) approximation, 9 one ignores the coupling terms in the second set of braces in $\mathrm{Eq}$. (7). Then the wave function in Eq. (5) may be represented by a single term with $\mu=\nu$ in the summation on the right side, i.e.,

$$
\psi_{\mu E}^{Q S H}=\left(R^{5 / 2} \sin \alpha \cos \alpha\right)^{-1} F_{\mu \mu E}(R) \phi_{\mu}\left(R ; \alpha, \hat{r}_{1}, \hat{r}_{2}\right) .
$$


For simplicity one usually sets $\mu=\nu$ and drops the double subscripts on $F$ when referring to the quasi-separable approximation solutions. One sees from Eq. (8) that the quasi-separable approximation amounts to assuming that motion in $R$ and motion in $\alpha$ are approximately independent of each other. This quasi-separability was inferred from Figs. 1 and 2, which show electron density plots obtained from quasi-separable approximation wave functions. This behavior may be confirmed by examining correlated two-electron wavefunctions and observing that the nodal lines of such wavefunctions also lie approximately along constant $R$ and along constant a. 14

It should be emphasized that although only single radial and angular functions are used to represent the two-electron wave function in Eq. (8), much electron correlation is implicitly included. This is illustrated in Fig. 3, which shows the $s^{2}\left({ }^{1} \mathrm{~S}\right), \mathrm{p}^{2}\left({ }^{1} \mathrm{~S}\right), \mathrm{d}^{2}\left({ }^{1} \mathrm{~S}\right)$, and $\mathrm{f}^{2}\left({ }^{1} \mathrm{~s}\right)$ components of the numerically calculated $\mathrm{H}^{-}\left({ }^{1} \mathrm{~S}\right)$ ground state angular function, $\phi_{\mu}$. One sees clearly that these higher angular momentum components are significant at small $\mathrm{R}$, near $\alpha \approx \pi / 4$ (i.e., $r_{1} \approx r_{2}$ ). As $\mathrm{R}$ increases, however, only the ss $\left.{ }^{1} \mathrm{~S}\right)$ component contributes significantly, in accordance with the independent electron model.

Notice also in Eq. (8) how all members of the channel $\mu$ have the same angular function $\phi_{\mu}$ at any given $R$. Each state of excitation energy $E$ within the channel $\mu$ is described by the radial function $\mathrm{F}_{\mu \mathrm{E}}$, which is calculated in the channel potential $\mathrm{U}_{\mu}(\mathrm{R})$ using $\mathrm{Eq}$. (7) and ignoring the off-diagonal coupling terms. Because each member of a Rydberg series of doubly excited states has the same angular function $\phi_{\mu}$ and has a radial function $\mathrm{F}_{\mu \mathrm{E}}(\mathrm{R})$ that is calculated in the same potential $U_{\mu}(R)$, the
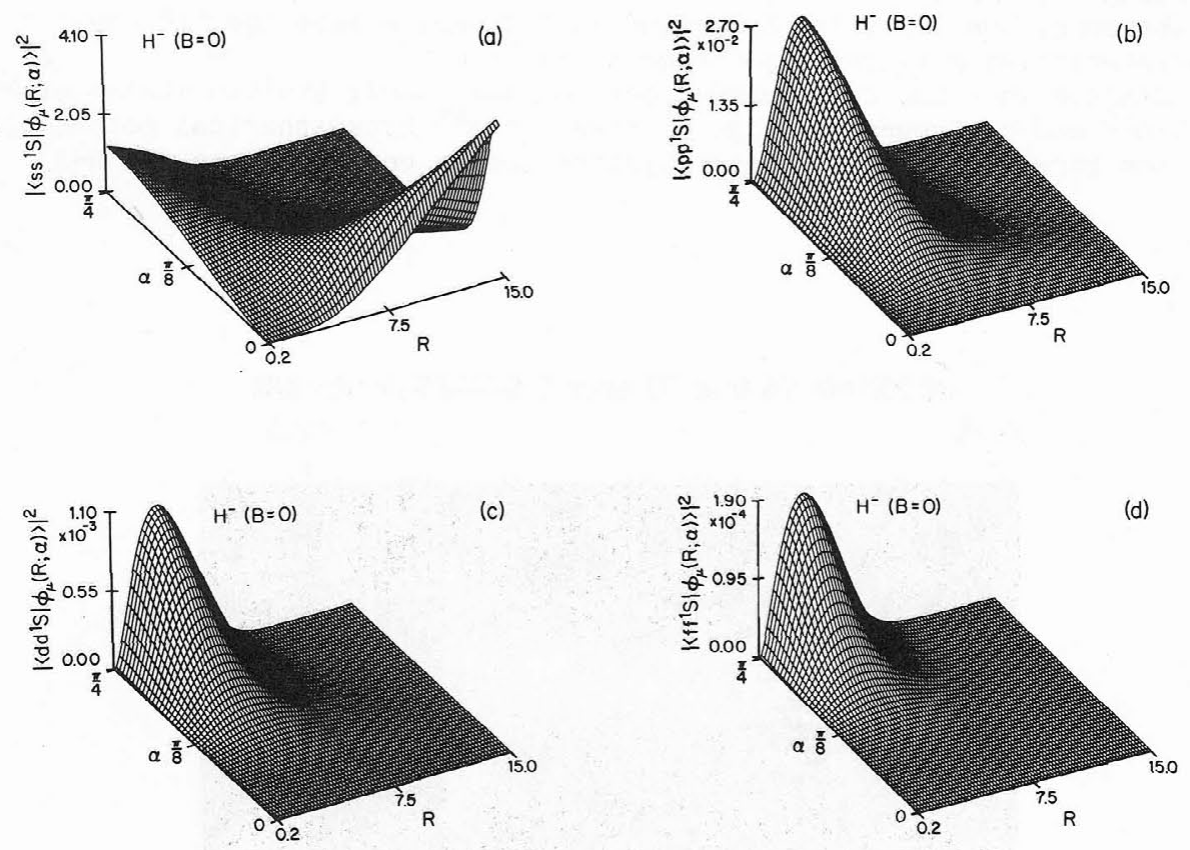

Fig. 3. Probability (per unit length in $\alpha$ ) for the angular momentum state $\ell \ell^{1} \mathrm{~S}$ in the singlet ground state of $\mathrm{H}^{-}$(a) $\ell=0 ;$ (b) $\ell=1 ;$ (c) $\ell=2$; (d) $l=3$. Note multiplication by factors of $10^{2}, 10^{3}, 10^{4}$ in Figs. (b), (c), and (d), respectively. (From Ref. 15) 
physical properties of states belonging to a particular channel $\mu$ are often immediately apparent upon examination of $U_{\mu}(R)$ and $\phi_{\mu}$. In what follows we illustrate the use of the potentials $U_{\mu}(R)$ to classify twoelectron excitation channels. We then survey the accuracy of numerical predictions obtained using the quasi-separable approximation.

\section{APPLICATIONS OF THE QUASI-SEPARABLE APPROXIMATION}

\section{Hyperspherical Classification of Two-Electron Excitation Channels}

The first major success 9 of the quasi-separable approximation in hyperspherical-coordinates was the classification and interpretation of the photoabsorption spectrum of $\mathrm{He}$ in the region of the doubly excited Rydberg states converging to the $n=2$ threshold. In the usual classification scheme there should be three Rydberg series of such levels of comparable intensity: $2 \mathrm{snp} 1 \mathrm{P}, 2 \mathrm{pnd}{ }^{1} \mathrm{P}$, and 2 pns $1 \mathrm{P}$. The experimental spectrum of Madden and Codling, 16 shown in Fig. 4, showed only one strong Rydberg series and one very weak Rydberg series. The third possible series was not observed. Cooper, Fano, and Prats ${ }^{17}$ interpreted the relative intensities of the two observed series in terms of the so-called "+" and "-" series, (2snp \pm 2 pns $)^{1} \mathrm{P}$. The "+" series members are more intense than those of the "-" series because the corresponding wavefunctions of the $"+"$ members have a much larger amplitude near the origin, allowing therefore a much larger overlap with the ground state. This scheme, however, does not explain the weakness of the 2 pnd ${ }^{1} \mathrm{P}$ channel. Fig. 5, however, shows Macek's hyperspherical potentials $U_{\mu}(R)$ for the three channels $\mu$ converging to the $n=2$ state of $\mathrm{He}^{+}$. One sees immediately that the three channels have vastly different centrifugal barriers near the origin, explaining the large intensity differences of the three allowed channels. Furthermore, the first two hyperspherical channels have the "+" and "-" characteristics predicted by Cooper et al. 17

Similar work has been carried out for the doubly excited states of $H$ by Lin 18 and by Greene. 19 Fig. 6 shows Lin's 18 hyperspherical potentials for the three $1 \mathrm{P}^{0}$ doubly excited Rydberg series converging to the $\mathrm{n}=2$

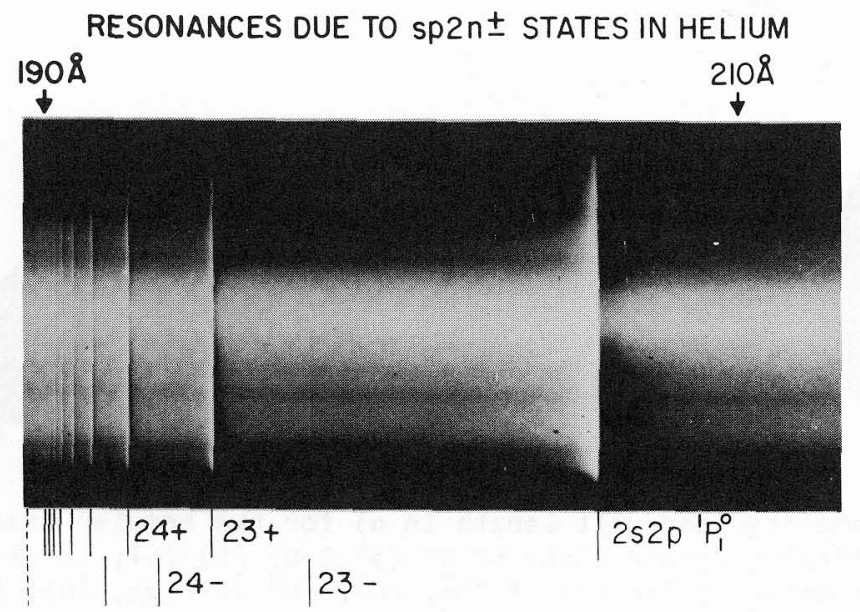

Fig. 4. Photoabsorption spectrum of He between 190 and $210 \AA$. The "+" and "-" series members are indicated below the spectrum. (From Ref. 16) 


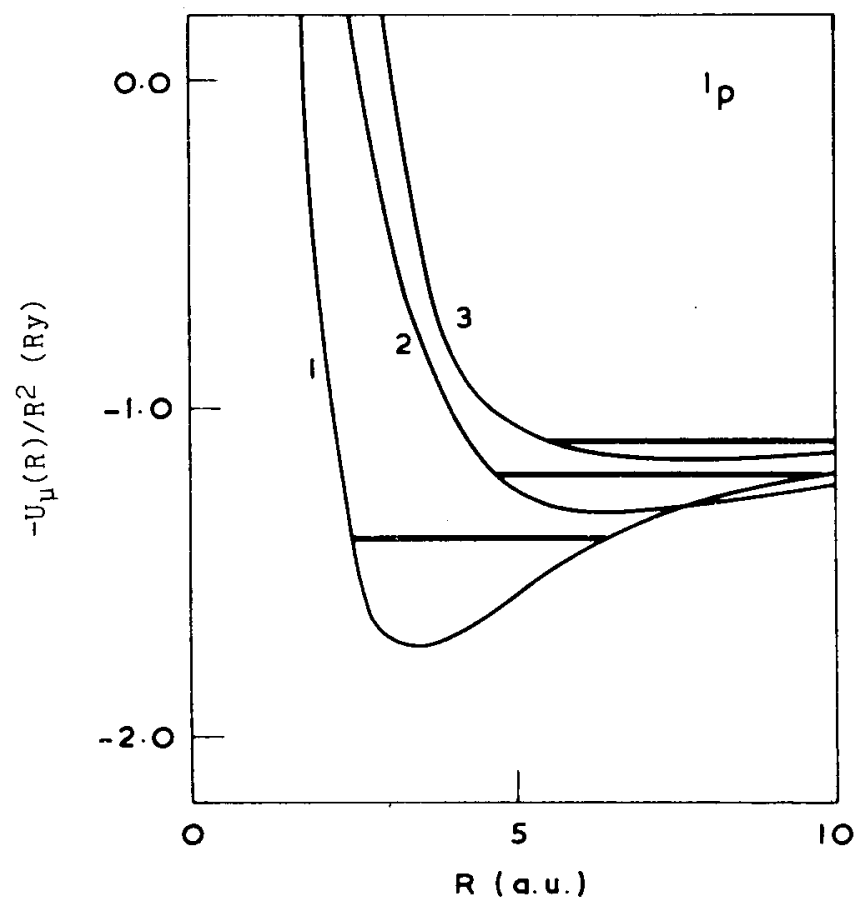

Fig. 5. Hyperspherical potential curves $-U_{\mu} / R^{2}$ vs. $R$ for the three He doubly excited ${ }^{i} \mathrm{P}$ channels converging to the $\mathrm{n}=2$ state of $\mathrm{He}^{+}$. (From Ref. 9)

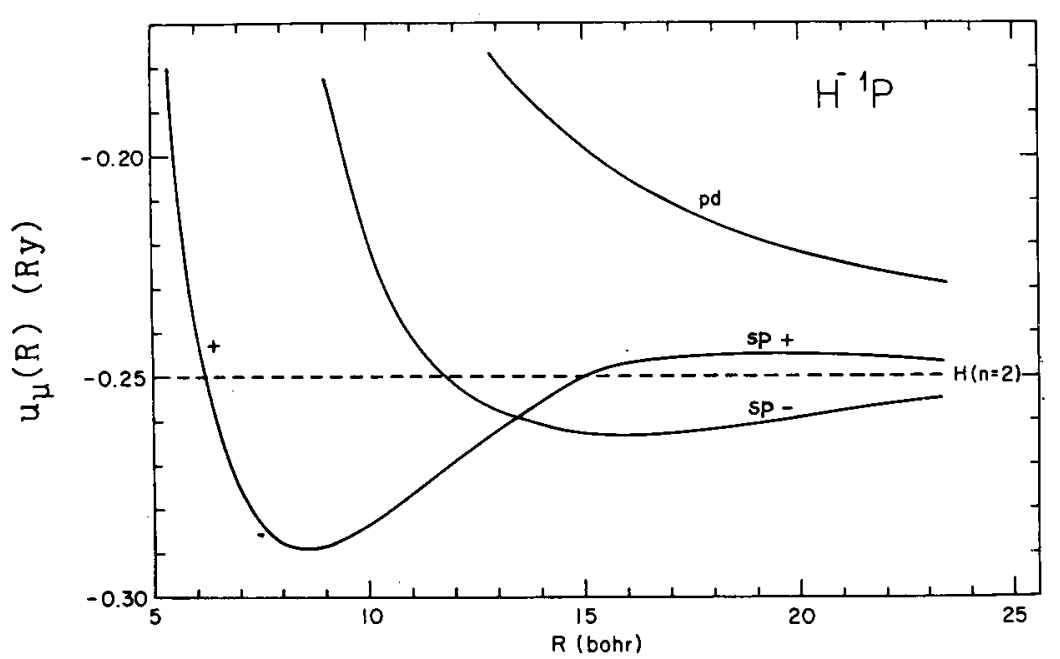

Fig. 6. Effective hyperspherical potential curves $u_{\mu}(R)$ vs. $R$ for the three $\mathrm{H}^{-}$doubly-excited ${ }^{1} \mathrm{P}$ channels converging to the $\mathrm{n}=2$ state of $H$. Note that $u_{\mu}(R) \equiv-\left[\left(U_{\mu}(R)+1 / 4\right) / R^{2}+\left(\phi_{\mu}, \frac{d^{2} \phi_{\mu}}{d R^{2}}\right)\right]$ (Cf. Eq. 7). (From Ref. 18) 
state of $H$. The "+" channel is repulsive at large $R$ and is not deep enough to support any bound states at small $R$. The repulsive barrier does, however, produce a shape resonance in this channel above threshold. The "-" potential is attractive at large $R$ and can support an infinity of Feshbach resonances. The "pd" channel is repulsive at all $R$ values. Fig. 7 shows Greene's hyperspherical potentials for the doubly excited $1 \mathrm{P}^{\circ}$ channels converging to the $n=3$ state of $H$. In this case the " + potential is always attractive and, since its centrifugal barrier is weaker than those of the other channels, the "+ series is the most strongly excited from the ground state. Greene used this hyperspherical channel calculation and quantum defect theory (QDT) to interpret the resonances obtained by Hamm et al.20 in the photodetachment spectrum of $\mathrm{H}^{-}$near the $\mathrm{n}=3$ threshold as due to the "+" series resonances. The data and Greene's QDT fit are in excellent agreement, as shown in Fig. 8.

\section{Energies, Phase Shifts, and Cross Sections}

The quasi-separable approximation in hyperspherical coordinates thus provides a very accurate qualitative description of Rydberg series of doubly excited states in $\mathrm{He}$ and $\mathrm{H}^{-}$. But how good are the quantitative predictions in this approximation? For the low values of the channel index, $\mu$, which have been studied, the answer depends on the excitation energy above the minimum in the hyperspherical potential $U_{\mu}$ of interest. For the lowest energy states calculated in the potentials $U_{\mu}(R)$, the quasi-separable approximation energies are in excellent agreement with experiment and with other theoretical results; the quasi-separable approximation wave function may also be used confidently. However, higher energy states of a particular channel $\mu$ calculated in the potential $U_{\mu}(R)$ are increasingly too high in energy, 9 if bound, or have too low phase shifts, $21-24$ if unbound. This is not surprising since for higher excitation energies the coupling between the hyperspherical channels can no longer be ignored. Thus at present the quasi-separable approximation in the hyperspherical coordinate approach provides a very good initial approximation to the exact electron wave function, but its systematic improvement for states of moderate and high excitation energy to provide state-of-the-art numerical predictions has been carried out in only a few cases.24-26 In what follows, we provide a few examples of the high level of accuracy to be expected from the quasi-separable approximation for the lowest excited

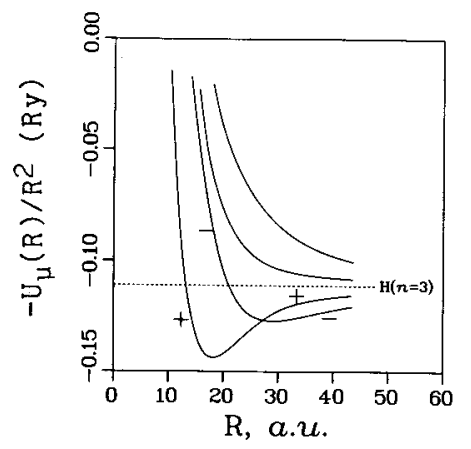

Fig. 7. Hyperspherical potential curves $-U_{\mu} / R^{2}$ vs. $R$ for $\mathrm{H}^{-}$doublyexcited $1_{\mathrm{P}}^{\mu}$ channels converging to the $n=3$ threshold of $H$. (From Ref. 19)

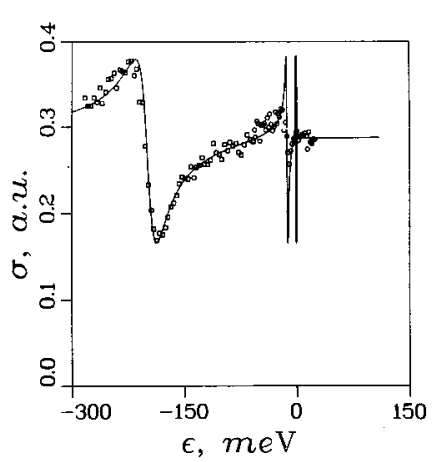

Fig. 8. Photodetachment cross section of $\mathrm{H}^{-}$near the $\mathrm{n}=3$ threshold of $\mathrm{H}$ at $\varepsilon=0$. Data: Hamm et al. (Ref. 20). Solid Line: QDT $f$ it of Greene (Ref. 19). (Erom Ref. 19) 
TABLE I. Elastic e-H ${ }^{1} \mathrm{~S}$ Scattering Phase Shift (in radians)a

\begin{tabular}{|c|c|c|c|c|}
\hline \multirow[b]{2}{*}{$\underline{k}\left(\underline{a}_{2} \underline{u}_{-}\right)$} & $\begin{array}{l}\text { Three- } \\
\text { Channel }\end{array}$ & \multicolumn{2}{|c|}{ Hyperspherical $^{\mathrm{C}}$} & \multirow{2}{*}{$\begin{array}{c}\text { "Exact" } \\
\text { Variational } \\
\text { Resultd }\end{array}$} \\
\hline & $\begin{array}{l}\text { Close- } \\
\text { Coupling }\end{array}$ & $\begin{array}{c}\text { One } \\
\text { Channel }\end{array}$ & $\begin{array}{c}\text { Two } \\
\text { Channel }\end{array}$ & \\
\hline 0.1 & 2.491 & 2.513 & 2.521 & 2.553 \\
\hline 0.2 & 1.974 & 1.983 & 2.023 & 2.067 \\
\hline 0.3 & 1.596 & 1.568 & 1.659 & 1.696 \\
\hline 0.4 & 1.302 & 1.242 & 1.380 & 1.415 \\
\hline 0.5 & 1.092 & 0.989 & 1.142 & 1.202 \\
\hline 0.6 & 0.93 & 0.784 & 0.926 & 1.041 \\
\hline 0.7 & 0.82 & 0.618 & 0.622 & 0.930 \\
\hline
\end{tabular}

a From Ref. 21, Table I.

b P.G. Burke and H.M. Schey, Ref. 30 .

C C.D. Lin, Ref. 21.

d C. Schwartz, Ref. 29 .

states in particular hyperspherical potentials.

Regarding level energies, Macek ${ }^{9}$ calculated the He $2 \mathrm{~s} 2 \mathrm{p}\left({ }^{1} \mathrm{p}\right)$ resonance excitation energy as $60.138 \mathrm{eV}$ as compared with the experimental value ${ }^{16}$ of $60.135 \pm .015 \mathrm{eV}$. Similarly, Miller and Starace 23 , calculated the ground state energy of He as -2.895 a.u. as compared with the essentially exact non-relativistic theoretical value 27 of $-2.904 \mathrm{a} . u$. Very recently Koyama et al.25 and Fukuda et al.26 have calculated energies for highlying doubly-excited states up to the $\mathrm{n}=9$ threshold in $\mathrm{H}^{-}$and up to the $\mathrm{n}=7$ threshold in He. They achieve agreement with results of other calculations within about $1 \%$ or so primarily by employing the diabatic 28 hyperspherical potentials in energy regions where the adiabatic (quasiseparable approximation) hyperspherical potentials have strongly avoided crossings. We shall discuss the use of diabatic hyperspherical potentials in the next section.

Regarding phase shifts, Lin ${ }^{21}$ calculated the e-H 1 S elastic scattering phase shift for values of electron momenta, $k$, in the range, $0 \leqq k$ (a.u.) $\leqq 0.7$. He used both the quasi-separable (one channel) hyperspherical approximation as well as a coupled two-channel hyperspherical approach. As shown in $\mathrm{Table} I$ for $k=0.1 \mathrm{a} . \mathrm{u}$. the quasi-separable approximation phase shift of 2.513 rad. is closer to the "exact" variational result of $2.553 \mathrm{rad}$. of Schwarti29 than is the three-channel close-coupling result of $2.491 \mathrm{rad}$. of Burke and Schey. 30 For $k \geqq 0.3$ a.u., however, the quasi-separable approximation results ${ }^{21}$ are lower than the three-channel close-coupling results 30 and both of these calculated predictions are lower than the variational results, 21 with the quasi-separable approximation results becoming increasingly too low as $k$ increases. Coupling two hyperspherical adiabatic (quasi-separable) channels improves the hyper- 
spherical predictions significantly, making them better than the threechannel close-coupling results up to $k=0.6$. However, only calculations which include 7 coupled hyperspherical channels, 24 shown in $\mathrm{Fig}$. 9 , have provided results equivalent to those of the variational method. 29

Lastly, regarding cross sections, a calculation 23 of the photoionization cross section of He using quasi-separable approximation hyperspherical coordinate wave functions demonstrates the strengths and weaknesses of the method. The initial and final wave functions for the process

$$
\mathrm{He}\left({ }^{1} \mathrm{~S}\right)+\gamma \rightarrow \mathrm{He}^{+} 1 \mathrm{~s}\left({ }^{2} \mathrm{~S}\right)+\mathrm{e}^{-(1 \mathrm{P})}
$$

both have the form of Eq. (8). For the initial state, $\mu$ corresponds to the lowest $1 S$ potential $U_{\mu}(R)$, and for the final state, $\mu$ corresponds to the lowest ${ }^{1} \mathrm{P}$ potential $\mathrm{U}_{\mu}(R)$. The photoionization cross section obtained using the quasi-separable approximation wave functions is shown in Fig. 10. Fig. 10 also shows the revised experimental results of Samson, 31 which have error bars of $\pm 3 \%$. The hyperspherical results lie within these error limits near threshold (for kinetic energies $0.0 \leqq \varepsilon \leqq 0.4$ a.u.) and in fact agree with experiment to within $1 \%$ at threshold. The hyper-

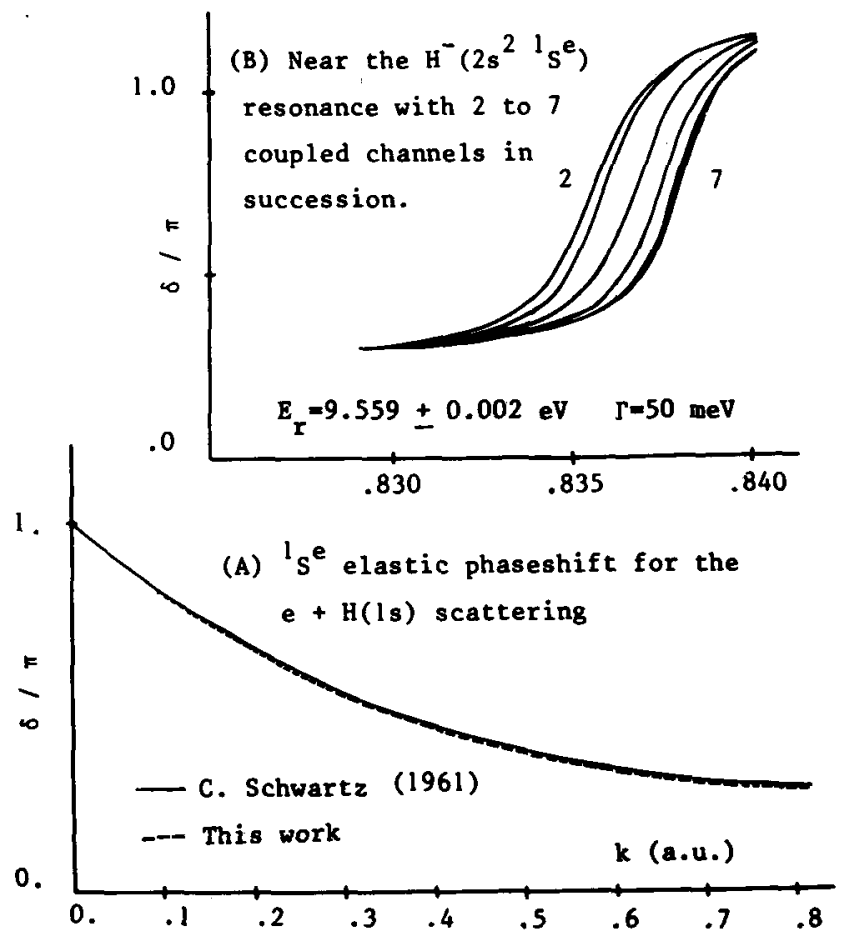

Fig. 9. Seven-channel hyperspherical calculation of the ' $\mathrm{S}^{\mathrm{a}}$ elastic scattering phase shift for electrons on $\mathrm{H}$ by L. Pelamourges, $\mathrm{S}$. Watanabe, and M. LeDourneuf, Ref. 24. (A) Phase shifts for electron momenta in the range, $0 \leqq k(a . u) \leqq$.0.8 . Solid lines shows comparison with variational results of $C$. Schwartz, Ref. 29 ; (B) Phase shifts for electron momenta in the range, $0.83 \leqq k($ a.u. $) \leqq 0.84$. (From Ref. 24) 
spherical results, however, are systematically lower than experiment above $\varepsilon=0.4 \mathrm{a} . \mathrm{u}$. Of the many other theoretical calculations, we show one with very good overall agreement with experiment: the four channel ( $1 \mathrm{~s}-2 \overline{\mathrm{s}}-2 \overline{\mathrm{p}})$ close-coupling calculation of Jacobs. 32 In comparison with the close-coupling results, the single-channel hyperspherical results are in better agreement with experiment below $\varepsilon=0.2 \mathrm{a} \cdot \mathrm{u}$. and are systematically lower above $\varepsilon=0.2 \mathrm{a} . \mathrm{u}$. Note that the length results of a more recent sixstate R-matrix calculation by Berrington et al. 33 are in excellent agreement with the experimental results of West and Marr, 34 including those near threshold, thereby indicating the sophistication required to properly describe the threshold region by methods employing independent electron representations.

\section{Discussion}

We see from these numerous examples that the quasi-separable approximation in hyperspherical coordinates provides an excellent first approximation for the representation of two-electron states. Simply from the potentials $U_{\mu}$ one can obtain much qualitative understanding of entire Rydberg series of two-electron excitations. Furthermore, the lowest energy states in each potential $U_{\mu}$ are well represented by quasi-separable approximation wave functions, as confirmed quantitatively by calculations of energies, phase shifts, and cross sections. A simple improvement on the quasi-separable approximation in regions of sharply avoided crossings of the potentials $U_{\mu}$ is to switch to a diabatic representation in these regions, as discussed in the next section. However, for energies far above the minima of the potentials $U_{\mu}$ of interest, quantitative accuracy requires the solution of the coupled equations (7). The few calculations which have been carried out indicate that the convergence of the calculated results proceeds slowly as the number of channels increases. Further analysis of this slow convergence is presented in the section after next.

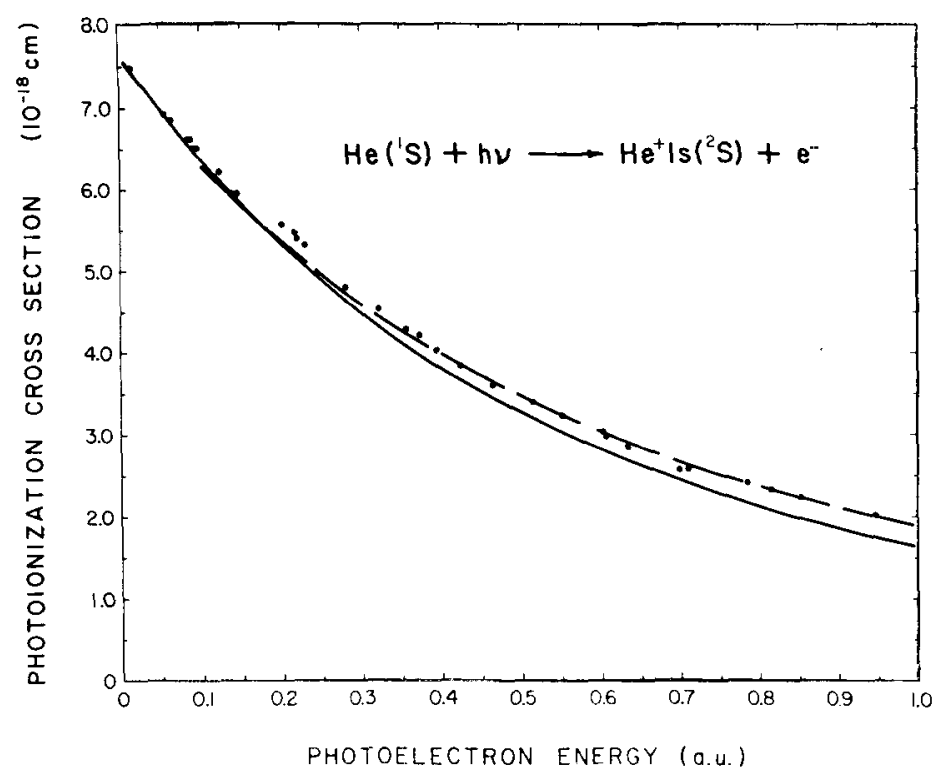

Fig. 10. Photoionization cross section for He. Full Curve: quasi-separable approximation (single channel) hyperspherical calculation of Miller and Starace (Ref. 23); Dots: Experimental results of Samson (Ref. 31); Dashed Curve: $1 \mathrm{~s}-2 \overline{\mathbf{s}}-2 \bar{p}$ (four channel) close-coupling calculation of Jacobs (Ref. 32). (From Ref. 23) 


\section{EVOLUTION OF TWO-ELECTRON EXCITATIONS}

\section{Analysis of the Angular Equation}

The hyperspherical coordinate approach has not only been used to study stationary states, but also to understand qualitatively how a low-energy two electron state concentrated near the origin evolves to states of high excitation far from the origin upon receiving energy during a collision process. The key idea, stressed by Fano 35 and illustrated graphically by Lin, 13 is that such states describe motion along a potential ridge centered about the direction $\alpha=\pi / 4$ (i.e., $r_{1}=r_{2}$ ).

Consider Eq. (6) for the channel functions $\phi_{\mu}\left(R ; \alpha, \hat{r}_{1}, \hat{r}_{2}\right)$. The potential $-C\left(\alpha, \theta_{12}\right)$, defined in Eq. (4), is shown in Fig. 11 for $Z=1$. States having one electron more excited than the other, i.e., $r_{2} \gg r_{1}$ or $r_{1} \gg r_{2}$, have an angle function $\phi_{\mu}$ with maximum amplitude in the valleys of the potential in Fig. 11, near $\alpha=0$ and $\alpha=\pi / 2$. Doubly-excited states of comparable energy have $r_{1} \approx r_{2}$ and thus the angle function $\phi_{\mu}$ for these states has maximum amplitude on the ridge of the potential in Fig. 11, near $\alpha=\pi / 4$, and preferably near $\cos \theta_{12}=-1$ (i.e., on opposite sides of the nucleus).

Consider now the $R$-dependence of the angle functions $\phi_{\mu}$. Eq. (6) shows that the potential $-C$ is multiplied by $R$. For large enough $R$, therefore, the potential -RC on the ridge becomes equal to the eigenvalue $U_{\mu}(R)$. At this "classical turning point" the angle function $\phi_{\mu}$ has no more "kinetic energy" of motion in $\alpha$ on the ridge. For larger $\mathrm{R}$ values, its amplitude on the ridge is exponentially damped and the probability amplitude in the channel $\mu$ must retreat to the valleys of the potential in $F i g .11, i m p l y^{-}$ ing that for such large $R$ values $\phi_{\mu}$ describes states with one electron more highly excited than the other. Alternatively, the two-electron state on the ridge may "hop" to the next higher channel $\mu^{\prime}$. With a higher value of $-U_{\mu}(R)$, the two electron excitation could move to somewhat larger $R$ along the ridge since the difference between $-U_{\mu}$, and the top of the potential ridge of $-\mathrm{RC}$ would restore some positive "kinetic energy" of motion in $\alpha$. Actually the vicinity of the classical turning point is

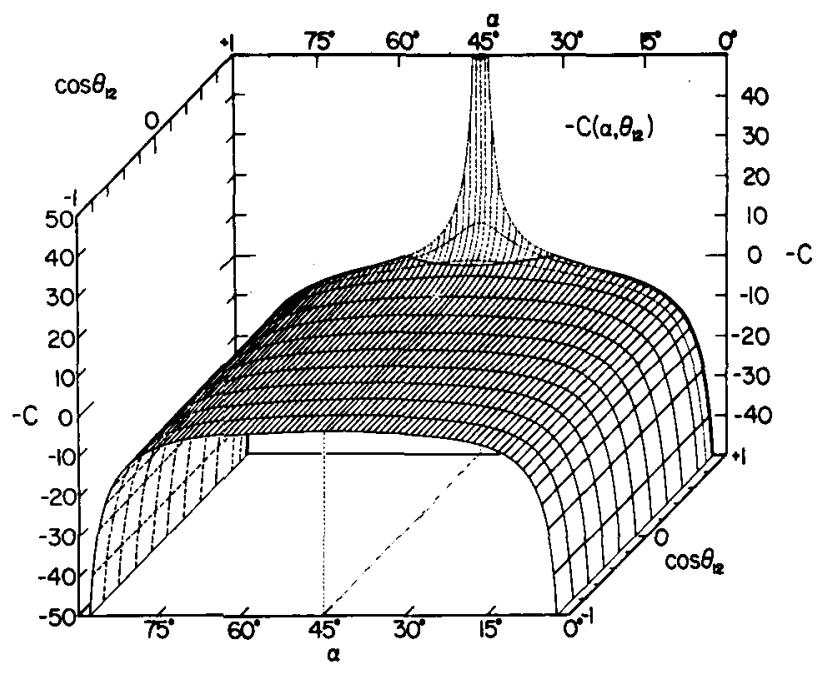

$z=1$

Fig. 11. Relief map of the potential $-C\left(\alpha, \theta_{12}\right)$ defined in Eq. (4) for $\mathrm{Z}=1$. (From Ref. 12) 
propitious for such a transition to a higher channel $\mu^{\prime}$ since the coupling matrix elements (cf. Eq. (7)) are largest precisely where the channel functions are changing most rapidly with $R$.

Lin 13 has shown graphically how the channel functions $\phi_{\mu}$ behave as functions of $\mathrm{R}$. In $\mathrm{Figs}$. 12 and 13 we show the $\mathrm{H}^{-}\left({ }^{1} \mathrm{~S}\right)$ channel functions $\phi_{\mu}\left(R ; \alpha, \theta_{12}\right)$ for $\mu=1$ and $\mu=2$ (i.e., the lowest two ${ }^{1} S$ hyperspherical channels). In Fig. 12 one sees that at $R=1$ the charge distribution in the first channel is peaked about $\alpha=\pi / 4$, lying on the potential ridge. At $R=4$, however, the charge distribution is vacating the ridge and moving to the valleys near $\alpha=0$ and $\alpha=\pi / 2$. By $R=8, \mu=1$ describes a channel with one electron much more highly excited than the other. Fig. 13 shows the next higher hyperspherical channel function. Note that at $R$ $=4$, precisely where $\mu=1$ has a depression along the ridge, the $\mu=2$ channel's charge distribution has a maximum. This peak in $\mu=2$ along the ridge progresses outward to larger $R$ values until at $R=12$ a depression appears along the ridge. If two-electron states in $\mu=2$ are to move to larger $R$ and remain comparably excited they must hop again to the next higher hyperspherical channel, and so on.

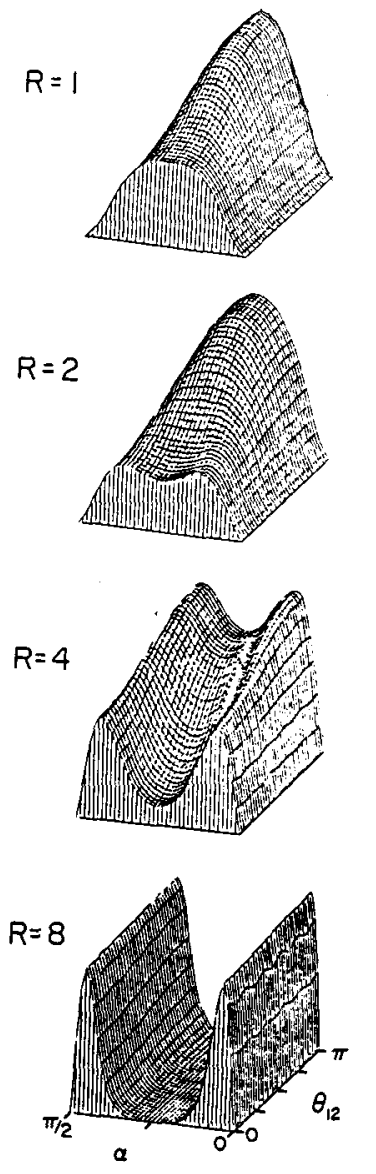

Fig. 12. Plot of $\left|\phi_{\mu}\left(R ; \alpha, \theta_{12}\right)\right|^{2}$ vs. $\alpha$ and $\theta_{12}$ for various $R$ values for the first $\mathrm{H}^{-}$is hyperspherical channel $\mu=1$. (From Ref. 13)
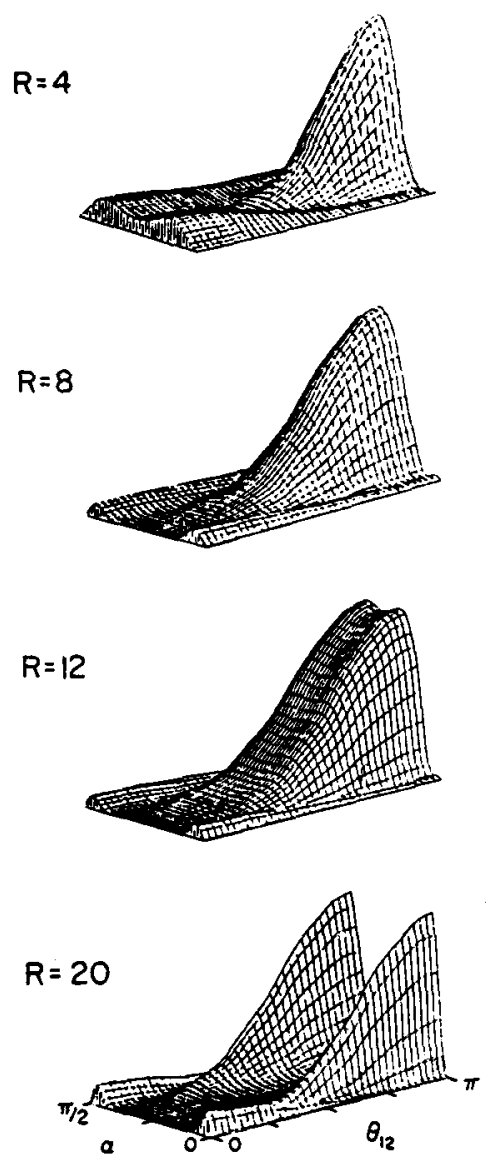

Fig. 13. Plot of $\left|\phi_{\mu}\left(R ; \alpha, \theta_{12}\right)\right|^{2}$ vs. $\alpha$ and $\theta_{12}$ for various $R$ values for the second $\mathrm{H}^{-}$1S hyperspherical channel, $\mu=2$. (From Ref. 13) 
This perspective of two electron excitation states evolving toward large radi $i \mathrm{R}$ along a potential ridge has its origins in the WannierPeterkop-Rau 36 analysis of electron impact ionization near threshold. Its application to quantitative predictions of excitation cross sections is just beginning. In particular, recent studies of photoionization of atoms having an outer $s^{2}$ subshell have shown some common features, which we now discuss.

\section{Adiabatic Vs. Diabatic Potentials}

Consider first the case of helium. As discussed above, the experimental observation of only a single intense Rydberg series converging to the $\mathrm{n}=2$ threshold in the photoabsorption spectrum of $\mathrm{He}$ can be understood easily in terms of the hyperspherical potentials shown in Fig. 5. The potential labelled "1" is the so-called "+" channel, whose states overlap the ground state much more effectively than do states in either the "2" or "-" potential or the "3" or "d" potential. Note however, that the "1" and "2" potentials cross at $R=7.64$.

Fig. 14, which examines this region in greater detail, shows that the adiabatic or quasi-separable approximation potentials actually do not cross, but have instead a sharply avoided crossing. Because of this avoided crossing over a small region in $\mathrm{R}$, the angle functions $\phi_{\mu}$ have large derivatives with respect to $R$. In fact, the coupling matrix elements are so large that the "+" and "-" potentials exchange their character for $R>7.64$. Fig. 15 shows this exchange by plotting the $R$-dependence of the overlap integral of $\phi_{-}(R=6.5)$ with $\phi_{-}(R)$ and with $\phi_{+}(R)$. Whereas for $R\left\langle 7.64,\left\langle\phi_{-}(6.5) \mid \phi_{-}(R)\right\rangle\right.$ is close to unity, as expected, and $\left\langle\phi_{-}(6.5) \mid \phi_{+}(R)\right\rangle$ is close to zero, one finds that for $\left.R\right\rangle 7.64$ $\left\langle\phi_{-}(6.5) \mid \phi_{+}(R)\right\rangle$ is close to unity and $\left\langle\phi_{-}(6.5) \mid \phi_{-}(R)\right\rangle$ is close to zero. What is happening is that electronic excitations populated in the " +" channel at small $R$ proceed outward at larger $R$ and "hop" from the quasiseparable "+" channel to the " $-"$ channel near $R=7.64$. For this reason one often employs the diabatic approximation shown in Fig. 14 in such

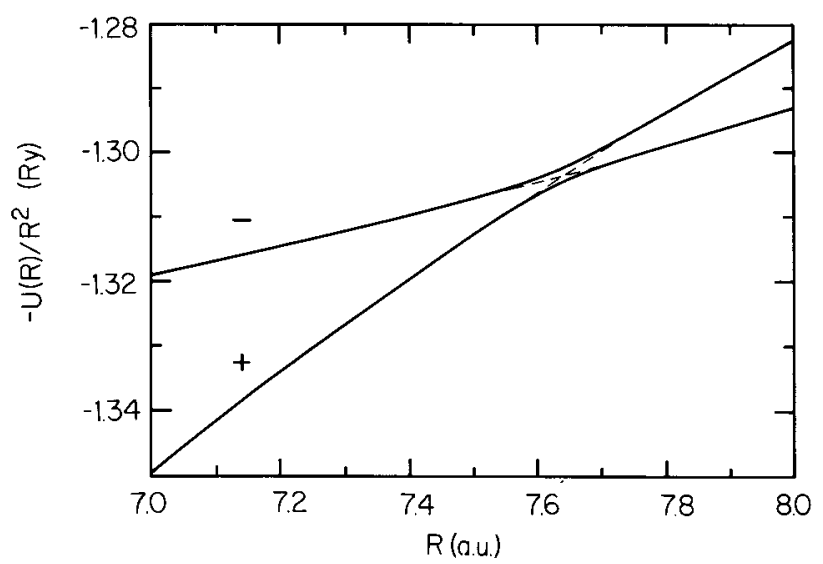

Fig. 14. The $\mathrm{He}^{*}(\mathrm{n}=2){ }^{1} \mathrm{P} "+" / "-"$ avoided crossing: a special case of transition from one $U_{\mu}(R)$ to another. Solid lines show the quasi-separable (adiabatic) hyperspherical potentials, which have an avoided crossing near $R=7.64$. The dashed Iines show the diabatic potentials, which cross near $R \approx 7.64$. 
cases of sharply avoided crossings. That is, one connects the "+" potential and channel function below $R=7.64$ to the "-" potential and channel function above $R=7.64$ and vice versa. One then ignores the residual coupling between the new "+" and "-" diabatic potentials.

\section{Photoionization of $\mathrm{Be}$}

Compare now this diabatic behavior observed in photoionization plus excitation of He leading to states converging to the $n=2$ threshold with photoionization of the outer $2 \mathrm{~s}^{2}$ subshell of beryllium.

Greene 37 has calculated the photoionization cross section of $\mathrm{Be}$ including coupling between the lowest two hyperspherical channels, $\mu=1$, corresponding to leaving the ion in its ground 2 s state, and $\mu=2$, corresponding to leaving the ion in its excited $2 \mathrm{p}$ state. (The inner s $^{2}$ core was represented by a central potential so that only the correlation of the outer two electrons was treated.) Greene's procedure is to calculate the two adiabatic potentials $U_{\mu}(R)$ and angle functions $\phi_{\mu}$ using the angular

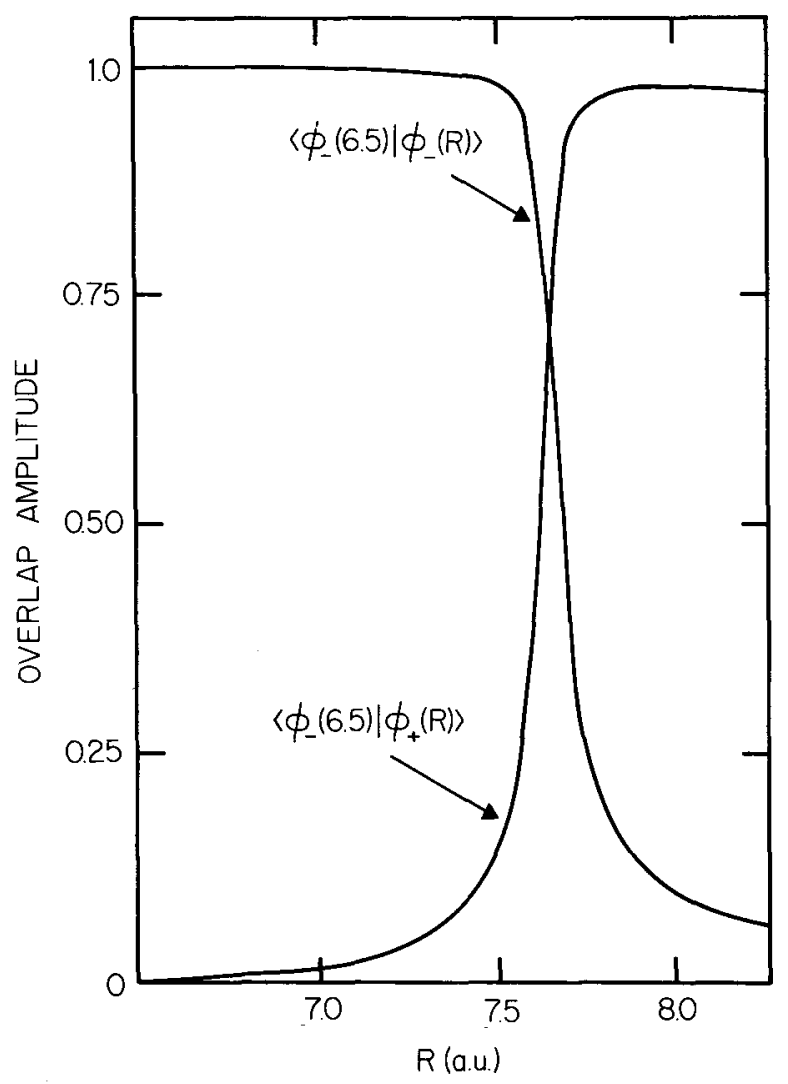

Fig. 15. Quasi-separable approximation channel function behavior at the "+"/" avoided crossing near $R=7.64$ as exhibited by the $R$-dependence of the two overlap integrals $\left\langle\phi_{-}\left(R=6.5 \mathrm{a}_{\text {. }} \mathrm{u}_{.}\right) \mid \phi_{-}(\mathrm{R})\right\rangle$ and $\left\langle\phi_{-}(R=6.5\right.$ a.u. $\left.) \mid \phi_{+}(R)\right\rangle$. Integrations are over $\alpha, \hat{r}_{1}$, and $\hat{r}_{2}$. 
Eq. (6). The radial Eq. (7) is then solved including the first and second derivative coupling matrix elements connecting the channels $\mu=1$ and $\mu=2$. His results are in reasonable agreement with the close-coupling calculation of Dubau and Wells 38 and show a very large intensity for excitation of the ion to the $2 p$ level.

The most interesting aspect of Greene's calculation 37 is the similarity his hyperspherical wave functions show to those in He, thereby indicating a similar behavior for $\mathrm{He}, \mathrm{Be}$, and all the alkaline earths. It is instructive first to compare the hyperspherical potentials $U_{\mu}(R)$ for the He ${ }^{1} \mathrm{P}$ levels converging to the $\mu=2$ threshold, shown in $\mathrm{Fig}$. 5 , to the corresponding potential curves in Be, shown in Fig. 16. One sees immediately from Fig. 5 why only one of the $\mathrm{He}^{+}(n=2)$ excitation channels, $\mu=$ 1 , is strongly populated; it has a much less repulsive potential barrier than either the $\mu=2$ or $\mu=3$ channels. Furthermore, the channel function $\phi_{\mu}$ for the $"+"$ channel $(\mu=1)$ is symmetric in $\alpha$, having an antinode on

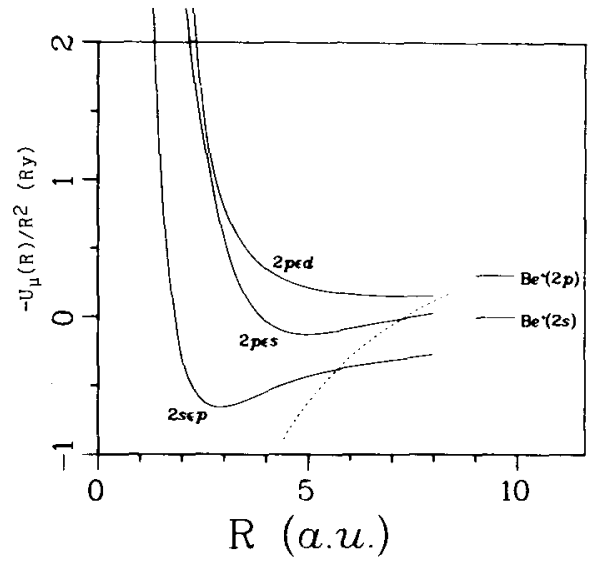

Fig. 16. Hyperspherical potential curves $-U_{\mu}(R) / R^{2}$ vs. $R$ for the three $B e$ $1 \mathrm{P}$ channels converging to the $\mathrm{n}=2$ state of $\mathrm{Be}^{+}$. (From Ref. 37)

the potential ridge in Fig. 11, while the "-" channel $(\mu=2)$ is antisymmetric in $\alpha$, having a node on the potential ridge. The symmetry about $\alpha=$ $\pi / 4$ for the $\mathrm{He}$ wavefunctions holds for all $\mathrm{R}$ values due to the degeneracy in energy of these channels. Reiterating the discussion above, recall that although the " + " and "-" channels are shown to cross in Fig. 5, this crossing is actually avoided; in any case the channel functions $\phi_{\mu}$ do not adjust to the crossing but proceed diabatically through it. For this reason the middle curve in Fig. 5 for $R>7.64$ a.u. has "+" character while the lowest curve for $R>7.64$ a.u. has "-" character.

Consider now the Be potentials in Eig. 16. Two differences from He are immediately apparent. First, the potential curves are non-degenerate for $\mathrm{R} \rightarrow \infty$. Secondly there is an avoided crossing between the first and second potential curves for $4<R<6$. Otherwise, however, one expects most of the absorption strength, as in $\mathrm{He}$, to go into the channel with the lowest potential curve. In his calculations Greene expanded the channel 


$$
\phi_{\mu}=\sum_{\ell_{1} \ell_{2}} g_{\mu}^{\ell_{1} \ell_{2}}(R ; \alpha) Y_{\ell_{1} \ell_{2} L M}\left(\hat{r}_{1}, \hat{r}_{2}\right)
$$

The most important functions $g_{\mu} l_{1} l_{2}$ (those with $l_{1} l_{2}=$ "sp") are shown in Fig. 17 for the potential curves $\mu=1$ and $\mu=2$ for various $R$ values. For $R=2$ one sees that the $\mu=1$ function is approximately symmetric about $\alpha=\pi / 4$ while the $\mu=2$ function is approximately antisymmetric, just as for the the "+" and "-" channels in He. As R increases, however, these adiabatic channel functions drop into one or the other of the potential valleys in Fig. 11, i.e., the $\mu=1$ amplitude becomes concentrated near $\alpha=0$ while the $\mu=2$ amplitude becomes concentrated near $\alpha=\pi / 2$. Thus, as $R$ increases, the non-degeneracy of the thresholds in Be causes a breakdown of the "+" and "-" symmetry about $\alpha=\pi / 4$ observed at small $R$ values. Furthermore, this transition is seen to occur for $R$ values $4 \leqq R \leqq 6$.

What is remarkable about Greene's treatment of the coupled radial equations (7) is the finding that the solution which at small $R$ starts out as the adiabatic wave function $F_{\mu=1}(R) \phi_{\mu=1}(R ; \Omega)$ in the $\mu=1$ channel becomes at $R>6$ a nearly equal superposition of the adiabatic wave functions for $\mu=1$ and $\mu=2$ in such a way that the "+" symmetry is preserved through the avoided potential crossing region. In other words, just as in He, the "+" solution proceeds diabatically through the avoided potential crossing. This also explains the large excitation cross section observed in Be since, unlike the case in He, the state having " + character becomes at R> 6 a nearly equal superposition of the $\mu=1$ and $\mu=2$ channel functions. Furthermore, it is expected that this diabatic behavior of the hyperspherical "+" solution will be a common feature of all alkaline earth and other similar two electron systems. 10,37

Indeed, R-matrix calculations 39 have found the eigenchannel functions for $\mathrm{Mg}{ }^{1} \mathrm{P}$ final states to be very similar in character to those for $\mathrm{Be}$. The heavier alkaline earth atoms $\mathrm{Ca}, \mathrm{Sr}, \mathrm{Ba}$, and $\mathrm{Ra}$ require the treatment of a still larger number of channels due to the proximity in energy of bound "d" orbitals. However, even for these elements, a hyperspherical
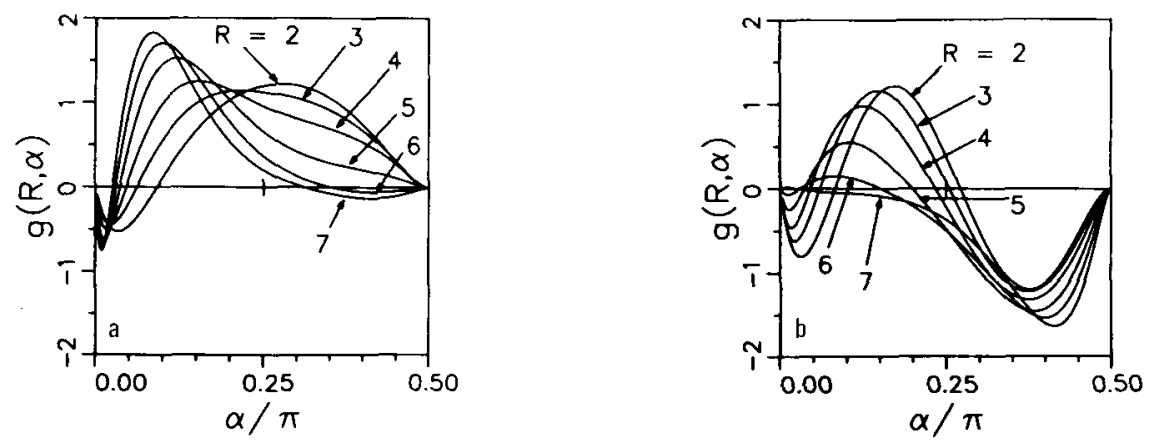

Fig. 17. Adiabatic "sp" channel wave functions associated with the lowest two Be potential curves: (a) $2 \mathrm{~s} \varepsilon \mathrm{p}, \mu=1$; (b) $2 \mathrm{p} \varepsilon s, \mu=2$. (From Ref. 37) 
analysis suggests that the diabatic character of a state populated at small $R$ is preserved as the state evolves toward larger $R, 10,37$ although, of course, this idea must yet be tested by carrying out the calculations. Very recently, R-matrix calculations for calcium 40 and strontium 41 have found that at small radial distances the wave functions and channel interactions "look remarkably similar for all of these atoms [He, $\mathrm{Be}, \mathrm{Mg}, \mathrm{Ca}$, Sr] including helium." $40(\mathrm{a})$ In any case, the discovery of the common features of photoexcitation processes in $\mathrm{He}, \mathrm{Be}$, and $\mathrm{Mg}$ as well as in the heavier alkaline earths, despite vast differences in the coupling strength between the associated channels, is one of the new perspectives on twoelectron correlations provided by the hyperspherical method. It indicates the usefulness of a combination of hyperspherical and R-matrix techniques to study electron correlations in these heavy atoms. 42

\section{HYPERSPHERICAL REPRESENTATIONS AT SMALL AND AT LARGE R}

A number of recent studies of hyperspherical wave functions at both large and small radii, $R$, have shed light on the strengths and weaknesses of the quasi-separable approximation to two-electron wave functions. We review briefly here three such studies.

\section{The Fock Expansion}

The proper series representation for the wave function of two electrons moving in the Coulomb field of a nucleus has been a long-standing problem that is still not completely solved. Both Bartlett and Fock independently introduced logarithmic terms in $\mathrm{R}$ into the expansion, although nowadays such expressions have come to be known as "Fock expansions." In particular, Bartlett 43 showed that an acceptable series solution of the Schrodinger equation for a two-electron atom or ion is the following series representation near the nucleus,

$$
\sum_{n, j} C_{n j} R^{n}(\operatorname{lnR}) j
$$

where $\mathrm{R}$ (Cf.Eq(1)) is the hyperspherical radius introduced by Gronwall. 44 In particular, series representations in terms of only the variables $r_{1}$, $r_{2}, r_{12} \equiv\left|r_{1}-r_{2}\right|$, and/or R--i.e., ignoring $1 n R$ terms - were shown not to exist. 43,45 Fock ${ }^{3}$ independently demonstrated that $\mathrm{Eq}$. (11) provides an acceptable series solution near the origin of the two-electron Schrödinger equation for ${ }^{1} \mathrm{~S}$ states. He also discussed in detail the procedure for calculating the coefficients $\mathrm{C}_{n j}$, which depend on five angular variables and hence obey rather complicated differential equations. Demkov and Ermolaev ${ }^{4}$ showed that Fock's expansion applies generally to an $\mathrm{N}$-electron wave function of arbitrary symmetry.

The usefulness of the series representation in Eq. (11) is dependent on its convergence properties. Macek 46 proved its convergence in the mean for values of $\mathrm{R}$ less than $(2|k|)^{-1}$, where the wave number, $k$, is related to the energy $E\left(a . u_{.}\right)$of the two-electron system relative to the double ionization threshold by

$$
E=k^{2 / 2}
$$

More recently, Morgan 47 has proved the convergence of the Fock expansion for $S$ states pointwise for all values of $R$ and has indicated that the expansion coefficients $C_{n j}$ are analytic functions of $E$. Yet to be proved 
is whether for discrete energies one of the solutions of the Schröinger equation having a convergent Fock expansion decays exponentially as either $r_{1}$ or $r_{2}$ tends to infinity, i.e., whether the "physical solutions" of the Schrödinger equation have Fock expansions. Leray 48 has begun to address this problem.

Although other studies of the Fock expansion have been carried out, the major application of Bartlett's and Fock's work has been to provide a better representation of the two-electron wave function in variational calculations.7,49,50 In particular, Frankowski and Pekeris 7 have shown that a 101-term variational trial function including $\ell$ nR terms gives better energies than a 1078-term variational trial function without lnR terms.

Very recently, theoretical interest has focused on determination of the exact two-electron wave function near the nucleus by explicit evaluation of the Fock coefficients, as opposed to the variational determination of a parameterized trial wave function including $\ell$ nR terms. Feagin, Macek, and Starace 51 have presented a detailed numerical procedure for the evaluation of the Fock coefficients of arbitrary order as expansions in hyperspherical harmonics. Abbott, Maslen, and Gottschalk52 have focused instead on analytic representations of the Fock coefficients, which they have obtained through second order in $\mathrm{R}$ for a state of arbitrary symmetry.

While the mathematical necessity of the logarithmic terms in Eq. (11) is acknowledged and the faster convergence their presence permits in variational calculations is known, 7 the physical consequences of these logarithmic terms remain unknown. Feagin, Macek, and Starace 51 attempted to determine this physical significance by matching numerically calculated Fock series solutions to quasi-separable approximation hyperspherical wave functions [cf. Eq. 8] at a boundary $R=R_{0}$ for ground and low-energy excited states of $\mathrm{He}$ and $\mathrm{H}^{-}$. Use of the Fock expansion for $\mathrm{R} \leqq \mathrm{R}_{0}$ did not significantly change the energies, wave functions, or phase shifts of the states considered from those calculated using the simple the quasi-separable approximation wave functions in hyperspherical coordinates over the entire range in $R$.

These calculations, 51 then, seem to indicate that for small $R$ the quasi-separable (adiabatic) approximation in hyperspherical coordinates is a very good representation of the lowest energy two-electron states. On the other hand, the quasi-separable radial wave functions do not have $\ln R$ terms in their series solution at $R=0$; an ordinary series expansion in powers of $R$ applies. It is only when coupling between quasi-separable channels is considered that $\ell n R$ terms are required to obtain a series solution of the full set of coupled equations (7). Future studies of processes for which the quasi-separable hyperspherical approximation is inadequate-such as excitation processes-are therefore required to identify the physical role played by the logarithmic terms in Eq. (11).

\section{Matching to Independent Particle Wave Functions}

In contrast to the apparent adequacy of the quasi-separable hyperspherical (QSH) wave function for two electron states at small $R$, as $R$ tends to infinity, i.e., as the electrons become separated, the QSH wave functions tend too slowly to the independent particle (IP) wave functions. Christiansen-Dalsgaard 53 has tackled this problem by matching the QSH wave functions to IP wave functions at finite values of $R$. For the case of $e^{--H}$ elastic scattering at low energies she has matched the QSH wave function corresponding to the $\mathrm{H}^{-}$ises( $\left.{ }^{1} \mathrm{~S}\right)$ channel $\mu$, i.e.,

$$
\psi^{\mathrm{QSH}}\left(\overrightarrow{\mathrm{r}}_{1}, \overrightarrow{\mathrm{r}}_{2}\right) \equiv \frac{\mathrm{F}_{\mu}(\mathrm{R}) \phi_{\mu}\left(\mathrm{R} ; \alpha, \hat{r}_{1}, \hat{r}_{2}\right)}{\left(\mathrm{R}^{5 / 2} \sin \alpha \cos \alpha\right)},
$$


to the IP wave function for this channel, i.e.,

$$
\psi\left(\vec{r}_{1}, \vec{r}_{2}\right) \equiv\left(\frac{P_{1 s}\left(r_{1}\right) P_{\varepsilon s}\left(r_{2}\right)+P_{1 s}\left(r_{2}\right) P_{\varepsilon S}\left(r_{1}\right)}{2^{1 / 2} r_{1} r_{2}}\right) Y_{00}\left(\hat{r}_{1}\right) Y_{00}\left(\hat{r}_{2}\right) .
$$

By requiring the logarithmic derivatives with respect to $R$ for these two wave functions to be equal at $R=R_{0}$, one may obtain the scattering phase shift of the IP one electron wave function, $P_{\varepsilon s}(r)$. Christensen-Dalsgaard chooses $R_{0}$ at each electron momentum $k$, defined by $\varepsilon=k^{2 / 2}$, so that exchange terms are negligible relative to direct terms in the IP representation.

The results of this matching procedure for the e-H elastic scattering phase shift are shown in Fig. 18. One sees that they are equivalent to the "exact" variational results of Schwartz up to the vicinity of the $2 s^{2}$ resonance energy. It achieves this accuracy by matching the best repre-

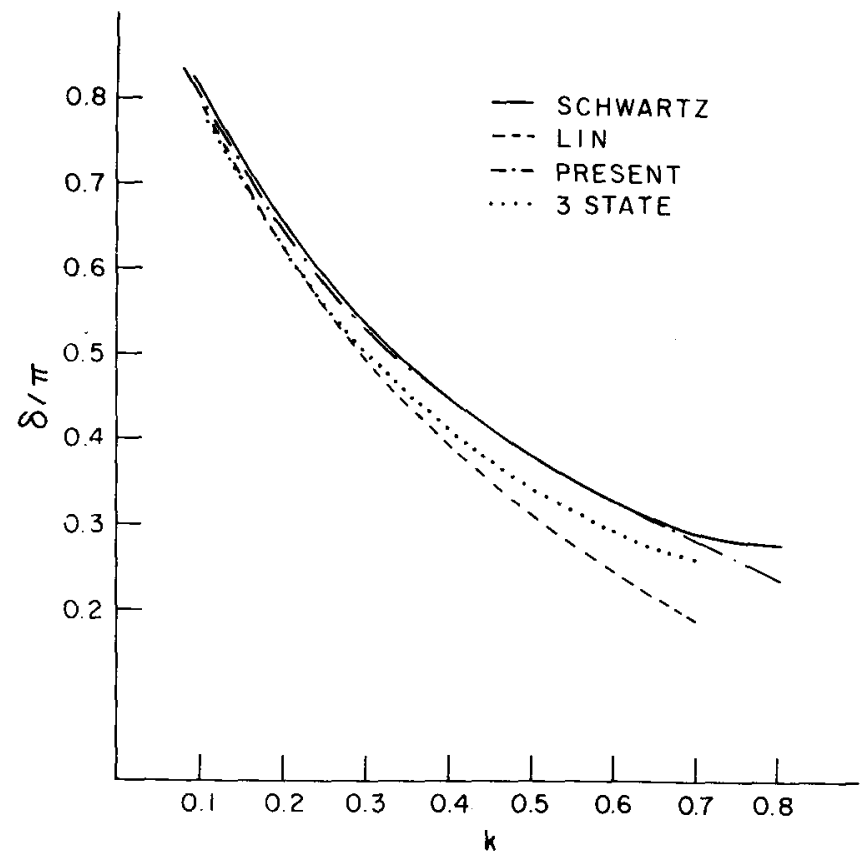

Fig. 18. Elastic scattering phase shifts, $\delta$, for electrons on $\mathrm{H}$ plotted vs. electron momentum, $k$. Solid Line: variational results of Schwartz, Ref. 29. Dashed Line: one-channel quasi-separable hyperspherical (QSH) results of Lin, Ref. 21. Dotted Line: three-channel close-coupling results

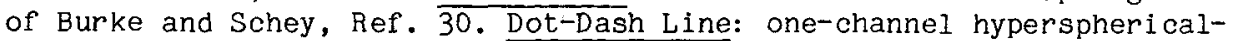
independent particle matching results of Christensen-Dalsgaard, Ref. 53 . (From Ref. 53). 
sentation at short distances (the QSH wave functions) to the best representation at large distances (the IP wave functions). The matching is done for values of $R$ in the range $6-8$ a.u. As shown in Fig. 3, this range in $R$ is transitional between electronic motion on the potential ridge (cf. Fig. 11) and electronic motion in the potential valleys (cf. Fig. 11).

The lessons provided by this calculation are that, firstly, the QSH wave functions are a very good representation at small distances where electronic motion is concentrated on the potential ridge; and secondly, when the wave function begins to depart from the potential ridge as $R$ increases, it is best to switch to an IP representation of the electronic motion.

\section{Asymptotic Expansions}

As shown by Macek, 9 the QSH wave functions do tend to an IP form at asymptotically large values of $R$. The work of Christensen-Dalsgaard, 53 however, has shown that the numerically calculated QSH wave functions should be matched to IP wave functions at much shorter distances in order to obtain accurate phase shifts and energies. To facilitate an analytic match, Zhen and Macek 54 have obtained asymptotic expansions in powers of $(1 / \mathrm{R})$ for QSH potentials and wave functions. The channel functions $\phi_{\mu}\left(R ; \alpha, \hat{r}_{1}, \hat{r}_{2}\right)$ have been determined through terms of order $R^{-2}$, and the potentials $U_{\mu}(R)$ through terms of order $R^{-4}$. Among their findings is that the QSH wave functions evolve into polarized orbitals as $R \sim \infty$.

\section{EXTENSIONS OF THE QUASI-SEPARABLE APPROXIMATION}

While the majority of the three-body systems studied by means of the quasi-separable approximation in hyperspherical coordinates have been twoelectron atomic and ionic systems in field-free space, a number of other three-body systems have been examined by this method in the last several years. These other three-body systems include the $\mathrm{H}_{2}{ }^{+}$molecule by Greene, 55 the $\mathrm{e}^{+}-\mathrm{H}$ system by Pelikan and Klar, $56 \mathrm{H}-$ in an electric field by $\mathrm{Lin}, 57 \mathrm{H}^{-}$and $\mathrm{He}$ in a magnetic field $\mathrm{B} \leqq 10^{9} \mathrm{G}$ by Park and Starace, 15 the positronium negative ion $\left(\mathrm{e}^{+}-\mathrm{e}^{-}-\mathrm{e}^{-}\right)$by Botero and Greene, 58 and the $\mathrm{HD}^{+}$ system by Macek and Jerjian. 59 In addition, Macek has used hyperspherical coordinates to study loosely bound states of three bosons interacting via short range two-body forces insufficient to bind any two of them. 60 One may expect further such extensions in the future.

\section{CONCLUSIONS}

We have surveyed broadly in this paper the use of the hyperspherical method to study three-body systems. In particular, we have shown that the quasi-separable (or adiabatic) approximation in hyperspherical coordinates provides a classification of doubly-excited electronic states, provides quantitatively accurate predictions for the lowest states in hyperspherical potentials and for processes connecting them, and provides at least qualitative insight into excitation processes. The evidence from several studies is that the quasi-separable wave functions, in general, provide an excellent representation for two electron wave functions at short distances near the nucleus, but tend too slowly to the more appropriate independent particle representation at large radial distances. Finally, we have mentioned a number of applications in recent years of the hyperspherical method to three-body systems other than two-electron atoms or ions in field-free space. 


\section{ACKNOWLEDGMENT}

This work has been supported in part by the U.S. Department of Energy, Division of Chemical Sciences, under Grant No. DE-FG 02-85ER13440.

\section{REFERENCES}

1. E.C. Kemble, The Fundamental Principles of Quantum Mechanics with Elementary Applications, (Dover Publications, Inc., New York, 1937) p. 210 .

2. P.M. Morse and H. Feshbach, Methods of Theoretical Physics (McGrawHill, New York, 1953) Vol. II, pp. 1730-1734.

3. V.A. Fock, Izv. Akad. Nauk USSR, Ser Fiz. 18, 161 (1954) [Eng. Transl.: Kong. Norske Videnskabers Selskabs Forh. 31, 138 (1958); 31, 145 (1958)].

4. Yu.N. Demkov and A.M. Ermolaev, Zh. Eksp. Teor. Fiz. 36, 896 (1959) [Sov. Phys.-JETP 36, 633 (1959)].

5. F.T. Smith, Phys, Rev. 120, 1058 (1960).

6. W. Zickendraht, Annals of Physics 35, 18 (1965).

7. (a) K. Frankowski and C.L. Pekeris, Phys. Rev. 146, 46 (1966);

(b) K. Frankowski, Phys. Rev. 160, 1 (1967);

(c) D.E. Freund, B.D. Huxtable, and J.D. Morgan, Phys. Rev. A 29, 980 (1984).

8. J.H. Macek, Phys. Rev. 160, 170 (1967).

9. J.H. Macek, J. Phys. B 2, 831 (1968).

10. U. Fano, Rep. Prog. Phys., 46, 97-165 (1983).

11. U. Fano and C.D. Lin, Atomic Physics 4 (Plenum, New York, 1975), pp. $47-70$.

12. C.D. Lin, Phys. Rev. A 10, 1986 (1974).

13. C.D. Lin, Phys. Rev. A 25, 76 (1982).

14. C.D. Lin, Phys. Rev. A 27, 22 (1983).

15. C.H. Park and A.F. Starace, Phys, Rev. A 29, 442 (1984).

16. R.P. Madden, and K. Codling, Astrophys. J. 141, 364 (1965).

17. J.W. Cooper, U. Fano, and F. Prats, Phys. Rev. Lett. 10, 518 (1963).

18. C.D. Lin, Phys. Rev. Lett. 35, 1150 (1975); Phys. Rev. A 14, 30 (1976).

19. C.H. Greene, J. Phys. B 13, L39 (1980). 
20. M.E. Hamm, R.W. Hamm, J. Donahue, P.A.M. Gram, J.C. Pratt, M.A. Yates, R.D. Bolton, D.A. Clark, H.C. Bryant, C.A. Frost, and W.W. Smith, Phys. Rev. Lett. 43, 1715 (1979).

21. C.D. Lin, Phys. Rev. A 12, 493 (1975).

22. H. Klar, and U. Fano, Phys. Rev. Lett. 37, 1132 (1976); H. Klar, Phys. Rev. A 15, 1452; H. Klar and M. Klar, Phys. Rev. A 17, 1007 (1978).

23. D.L. Miller and A.F. Starace, J. Phys. B 13, L525 (1980).

24. L. Pelamourges, S. Watanabe, and M. LeDourneuf, in Electronic and Atomic Collisions: Abstracts of Contributed Papers, XIII ICPEAC, Edited by J. Eichler, W. Fritsch, I.V. Hertel, N. Stolterfoht, and U. wille (Berlin, 1983), p. 109.

25. N. Koyama, H. Fukuda, T. Motoyama, and M. Matsuzawa, J. Phys. B 19, L331 (1986).

26. H. Fukuda, N. Koyama, and M. Matsuzawa, J. Phys. B 20, 2959 (1987).

27. C.L. Pekeris, Phys. Rev. 112, 1649 (1958).

28. See, e.g., B.L. Christiansen-Dalsgaard, Phys. Rev. A 29, 470 (1984). See also the lecture by U. Fano, "General Features of Collisions and spectra," in this volume.

29. C. Schwartz, Phys. Rev. 124, 1468 (1961).

30. P.G. Burke, and H.M. Schey, Phys. Rev. 126, 147 (1962).

31. J.A.R. Samson, Phys. Reports 28C, 303 (1976).

32. V.L. Jacobs, Phys. Rev. A 3, 289 (1971).

33. K.A. Berrington, P.G. Burke, W.C. Fon, and K.T. Taylor, J. Phys. B 15, L603 (1982).

34. J.B. West and G.V. Marr, Proc. Roy. Soc. A 349, 397 (1976).

35. U. Fano, Phys. Rev. A 22, 2660 (1980).

36. G. Wannier, Phys. Rev. 90, 817 (1953); R. Peterkop, J. Phys. B 4, 513 (1971); A.R.P. Rau, Phys. Rev. A 4, 207 (1971).

37. C.H. Greene, Phys. Rev. A 23, 661 (1981).

38. J. Dubau and J. Wells, J. Phys. B 6, 1452 (1973).

39. P.F. O'Mahony and C.H. Greene, Phys. Rev. A 31, 250 (1985).

40. (a) C.H. Greene and L. Kim, Phys. Rev. A 36, 2706 (1987).

(b) L. Kim and C.H. Greene, Phys. Rev. A 36, 4272 (1987).

41. M. Aymar, E. Luc-Koenig, and S. Watanabe, J. Phys. B 20, 4325 (1987). 
42. P.F. O'Mahony and S. Watanabe, J. Phys. B 18, L239 (1985).

43. J.H. Bartlett, Phys. Rev. 51, 661 (1937).

44. T.H. Gronwall, Phys. Rev. 51, 655 (1937).

45. J.H. Bartlett, J.J. Gibbons, and C.G. Dunn, Phys. Rev. 47, 679 (1935).

46. J.H. Macek, Phys. Rev. 160, 170 (1967).

47. J.D. Morgan, Theor. Chim. Acta. 69, 181-223 (1986).

48. J. Leray, in Lecture Notes in Physics 195: Trends and Applications of Pure Mathematics to Mechanics, Edited by P.G. Ciarlet and M. Roseau (Springer, Berlin, 1984), pp. 235-247.

49. A.M. Ermolaev and G.B. Sochilin, Dokl. Akad. Nauk SSSR 155, 1050 (1964) [Sov. Phys.-Dokl. 9, 292 (1964)].

50 G.O. Morrell and D.L. Knirk, Theor. Chim. Acta 37, 345 (1975).

51. J.M. Feagin, J. Macek, and A.F. Starace, Phys. Rev. A 32, 3219 (1985).

52. (a) P.C. Abbott and E.N. Maslen, J. Phys. A 20, 2043 (1987).

(b) J.E. Gottschalk, P.C. Abbott, and E.N. Maslen, J. Phys. A 20, 2077 (1987).

53. B.L. Christensen-Dalsgaard, Phys. Rev. A 29, 2242 (1984).

54. Z. Zhen and J. Macek, Phys. Rev. A 34, 838 (1986).

55. C.H. Greene, Phys. Rev. A 26, 2974 (1982).

56. E. Pelikan and H. Klar, Z. Phys. A 310, 153 (1983).

57. C.D. Lin, Phys. Rev. A 28, 1876 (1983).

58. (a) J. Botero and C.H. Greene, Phys. Rev. A 32, 1249 (1985).

(b) J. Botero, Phys. Rev. A 35, 36 (1987).

59. J. Macek and K.A. Jerjian, Phys. Rev. A 33, 233 (1986).

60. J. Macek, Z. Phys. D 3, 31 (1986). 